\title{
Increasing $\mathrm{NAD}^{+}$availability in skeletal muscle to
}

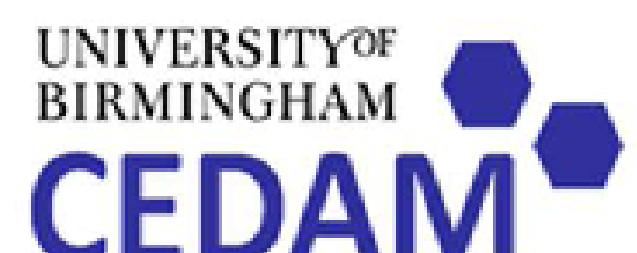

\section{augment energy metabolism}

Rachel Fletcher, Craig Doig, Agnieszka Zielinska, Aliesha Griffin, Andrew Philp \& Gareth Lavery.

Centre for Endocrinology, Diabetes and Metabolism, University of Birmingham, Edgbaston, Birmingham, B15 2TT, UK

\section{Background}

$\mathrm{NAD}^{+}$, an essential coenzyme in energy production, has recently risen to prominence as a signalling molecule central in mediating cellular metabolism and mitochondrial function. $\mathrm{NAD}^{+}$dependent protein deacetylase sirtuin (SIRT) proteins regulate key metabolic transcription factors, including FOXOs and PGC-1 $\alpha$ in muscle in response to cellular energy demands and metabolic stress (1). Declining $N A D^{+}$, metabolic and mitochondrial function are hallmark features of many patho-physiological processes such as ageing and type 2 diabetes (2). Thus, boosting NAD+ availability may have beneficial and therapeutic potential. $\mathrm{NAD}^{+}$ consumption (e.g. SIRTS) requires its re-synthesis through precursor salvage to maintain appropriate levels.

Here we identify important NAD+ salvage pathways in skeletal muscle that could be utilised to 'boost' NAD+ levels to support energy homeostasis during metabolic decline and stress (Fig. 1). Furthermore, we test the potential of salvageable NAD+ precursors to modulate skeletal muscle mitochondrial function.

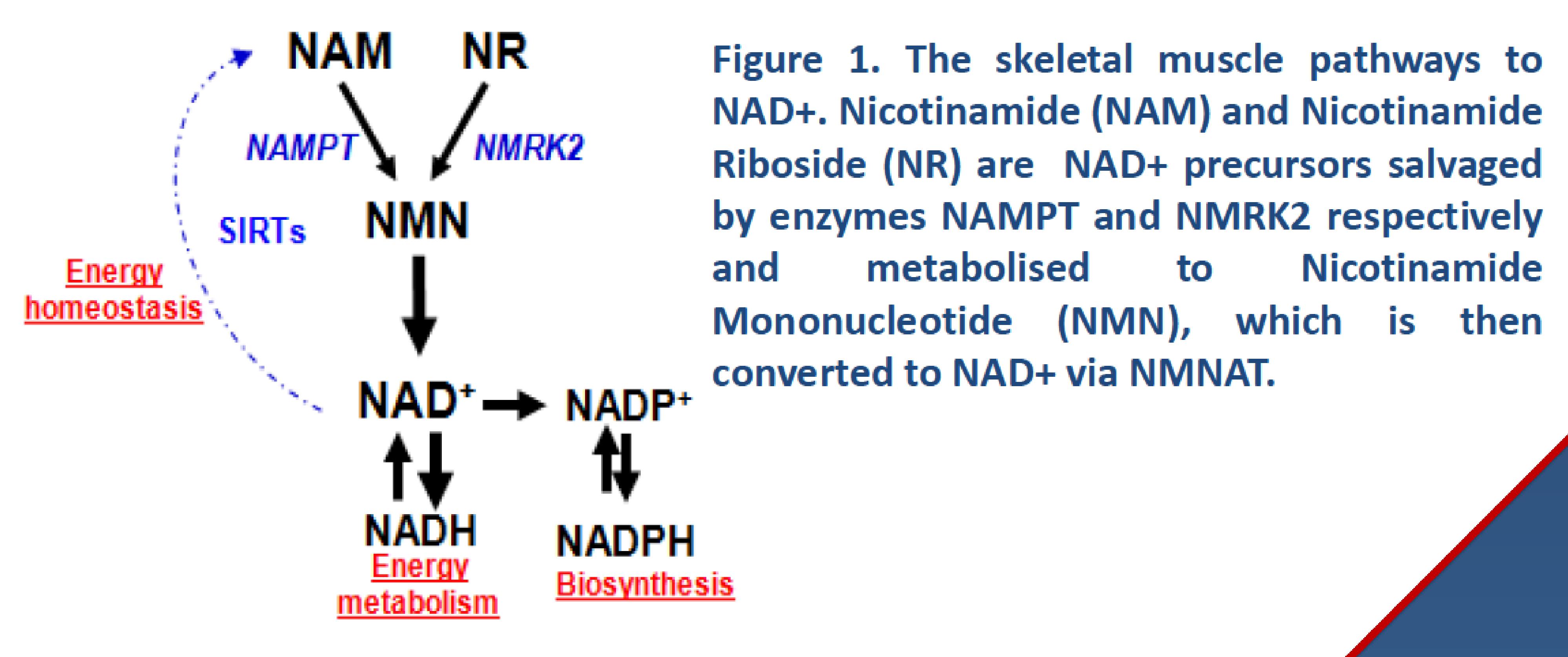

\section{Experimental design}

- Identify the NAD+ biosynthesis genes in skeletal muscle

Use C2C12 muscle cells as an in vitro model for manipulation of cellular NAD+ content (e.g. Precursor supplementation, enzyme inhibition)

- Use Nmrk2 KO muscle to characterise phenotype, harvest muscle tissue and isolate satellite cells for culture of primary myotubes (Fig.2).

\section{Results}

\section{Characterisation of mouse muscle NAD+ salvage pathways}

Nmrk2 and Nampt are the major NAD+ skeletal muscle biosynthesis pathways

$$
\text { 3.A. }
$$

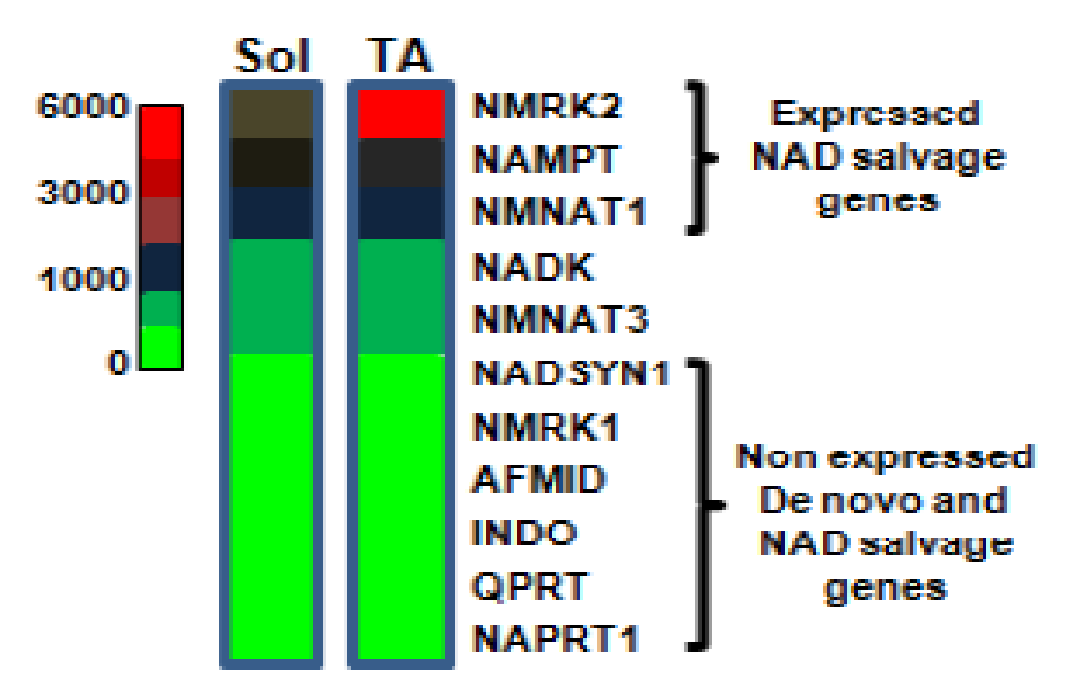

B.

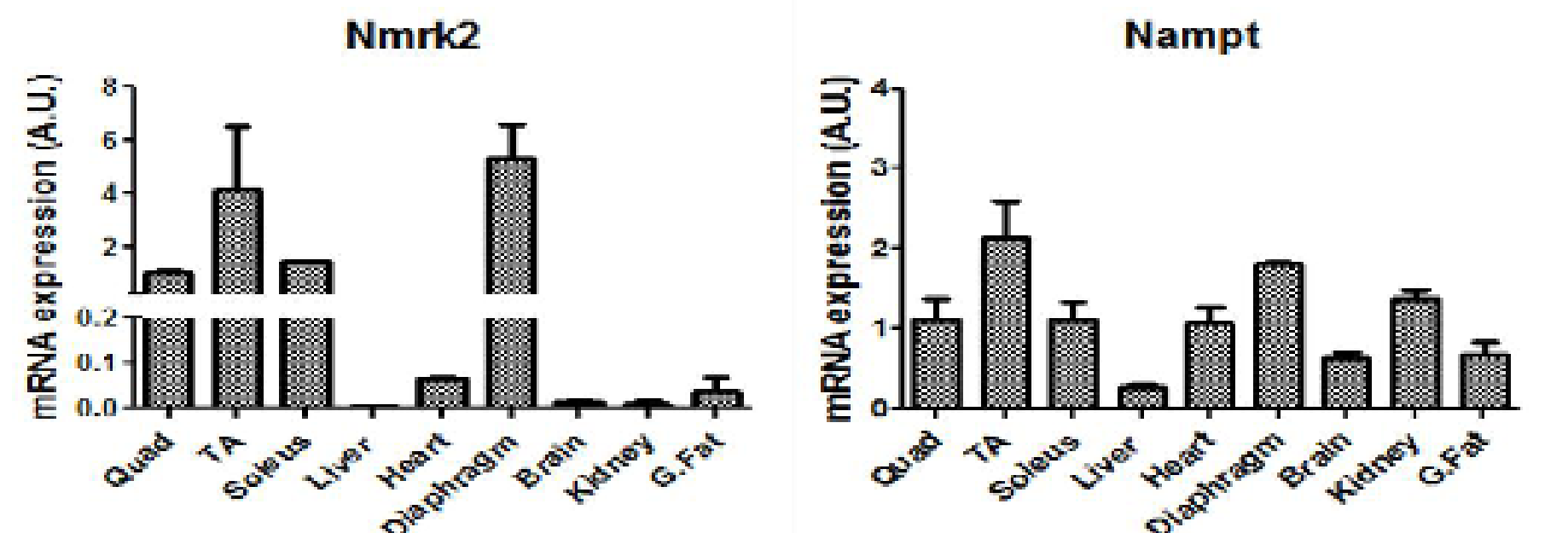

Nmrk2 is switched on during muscle development

$$
\text { c. }
$$

